\title{
Miocardiopatía chagásica en España: un diagnóstico a tener en cuenta
}

\author{
F. FLORIÁN SANZ, C. GÓMEZ NAVARRO, N. CASTRILLO GARCÍA', A. PEDROTE \\ MARTÍNEZ, E. LAGE GALLÉ
}

Servicio de Cardiología. 'Servicio de Medicina Interna. Hospital Universitario Virgen del Rocío. Sevilla

\begin{abstract}
RESUMEN
La enfermedad de Chagas es un importante problema de salud en Latinoamérica y una de las causas secundarias de miocardiopatía dilatada. La miocardiopatía chagásica crónica es su consecuencia más devastadora y principal causa de mortalidad. Las migraciones de poblaciones rurales hacia las ciudades y al extranjero han modificado su epidemiología clásica, haciendo posible su presencia en Estados Unidos y Europa. Presentamos el caso de un paciente que ingresó en situación de insuficiencia cardiaca congestiva y que fue diagnosticado de miocardiopatía chagásica crónica. Hasta donde conocemos, es el primer caso en la bibliografía de una miocardiopatía dilatada de etiología chagásica en España.
\end{abstract}

PALABRAS CLAVE: Insuficiencia cardiaca. Miocardiopatía dilatada. Enfermedad de Chagas.
CHAGASIC CARDIOMYOPATHY IN SPAIN: A DIAGNOSIS TO BEAR IN MIND

\section{ABSTRACT}

Chagas' disease is an important public health problem in Latin America and one of the specific causes of dilated cardiomyopathy. Chronic chagasic cardiomyopathy is the worse result of this disease and its main cause of mortality. Rural migrations from endemic to nonendemic countries make possible observing affected patients in United States and Europe. We report the case of a patient with congestive heart failure who was diagnosed with chronic chagasic cardiomyopathy. This is the first case of chagasic dilated cardiomyopathy in Spain.

KEY WORDS: Heart failure. Dilated cardiomyopathy. Chagas' disease.

Florián Sanz F, Gómez Navarro C, Castrillo García N, Pedrote Martínez A, Lage Gallé E. Miocardiopatía chagásica en España: un diagnóstico a tener en cuenta. An Med Interna (Madrid) 2005; 22: 538-540.

\section{INTRODUCCIÓN}

La combinación de dilatación y disfunción sistólica del ventrículo izquierdo o de ambos ventrículos, de origen idiopático o específico, define a la miocardiopatía dilatada. Constituye una causa importante de morbimortalidad cardiovascular por insuficiencia cardiaca congestiva o por arritmias, siendo el diagnóstico más frecuente en pacientes sometidos a trasplante cardiaco (1). Aunque la mayoría de los casos son de origen idiopático, puede estar causada por una gran diversidad de enfermedades específicas, algunas de las cuales pueden ser potencialmente reversibles. Entre las causas secundarias figuran tóxicos, enfermedad coronaria, miocarditis, alteraciones endocrinas y enfermedades infecciosas.

La enfermedad de Chagas está causada por el parásito protozoario Trypanosoma cruzi y se transmite al humano habitualmente por insectos hematófagos del género Triato- $m a$, aunque también por vía materno-fetal o mediante transfusión sanguínea. De 16 a 18 millones de personas están infectadas en América Latina y unos 120 millones están en riesgo de infectarse $(2,3)$. Aproximadamente el $30 \%$ de los infectados desarrollan una forma crónica de la enfermedad, caracterizada por daño miocárdico (insuficiencia cardiaca, diversas arritmias, fenómenos embólicos) y/o alteraciones digestivas (megacolon, megaesófago). Debido a las características actuales de la inmigración en nuestro país, debemos incluirla dentro de las posibles causas secundarias de miocardiopatía dilatada en los pacientes procedentes de áreas endémicas.

A continuación describimos el caso de un paciente que ingresó en situación de insuficiencia cardiaca congestiva y que fue diagnosticado de miocardiopatía chagásica crónica. Constituye el primer caso documentado en España de una miocardiopatía dilatada de etiología chagásica. 

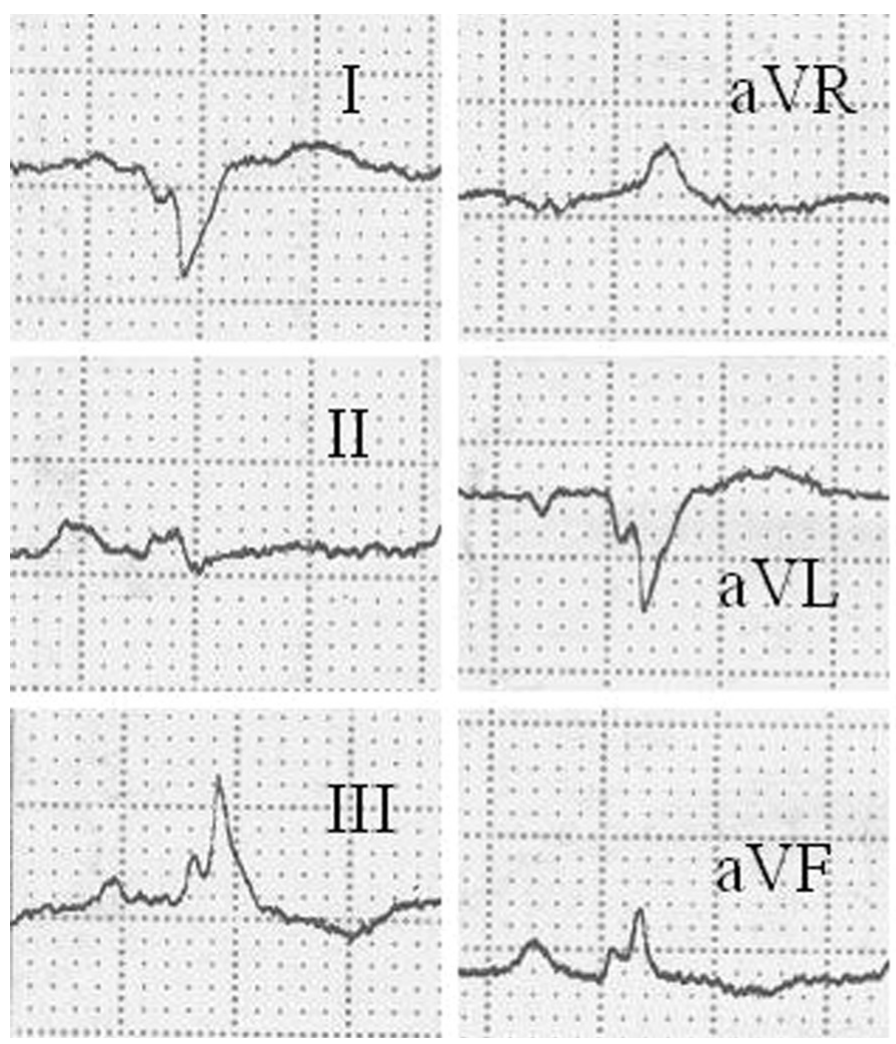

Fig. 1. Electrocardiograma basal.

\section{CASO APORTADO}

Varón de 48 años, natural de Bolivia y residente en España desde hacía dos años, sin hábitos tóxicos ni factores de riesgo cardiovascular conocidos. Fue ingresado en nuestro centro por presentar disnea de esfuerzo de aparición en las últimas tres semanas y que había progresado hasta hacerse de reposo.

A la exploración física a su ingreso, destacaban una presión arterial de 110/75 mmHg, taquipnea en reposo, ingurgitación yugular y hepatomegalia. En la auscultación se apreciaban unos tonos cardiacos rítmicos y sin soplos a 90 latidos por minuto y crepitantes en campos medios y bases pulmonares.

El electrocardiograma mostraba un ritmo sinusal, con eje desviado a la derecha y bloqueo completo de rama derecha (Fig. 1). En la radiografía de tórax presentaba cardiomegalia y signos de éstasis pulmonar. Las determinaciones de bioquímica sérica, marcadores cardiacos, perfil hepático, lípidos, hormonas tiroideas, hemograma y estudio de coagulación fueron normales. El ecocardiograma transtorácico mostró un ventrículo izquierdo dilatado (diámetro telediastólico de $65 \mathrm{~mm}$ ), con aquinesia posteroinferior e hipoquinesia del resto de segmentos y fracción de eyección severamente deprimida (20\%). Presentaba regurgitación mitral ligera con un patrón de llenado de fisiología restrictiva, regurgitación tricuspídea e hipertensión pulmonar moderadas y dilatación de aurícula izquierda y de cavidades derechas (Fig. 2). El estudio Holter de 24 horas mostró una alta carga de extrasistolia ventricular, con algunos eventos de bigeminismo aislados y sin episodios de taquicardia ventricular.

Ante el diagnóstico de miocardiopatía dilatada, se inició estudio para descartar causas secundarias de la misma. En el cateterismo cardiaco, las arterias coronarias fueron angiográficamente normales. Los principales resultados de los estudios serológicos llevados a cabo se muestran en la tabla I. Debido al origen del paciente, se consideró la posibilidad de una enfermedad de Chagas, por lo que se enviaron muestras sanguíneas al Centro Nacional de Microbiología. La serología positiva para Tripanosoma cruzi (IFI título IgG 1/320 y
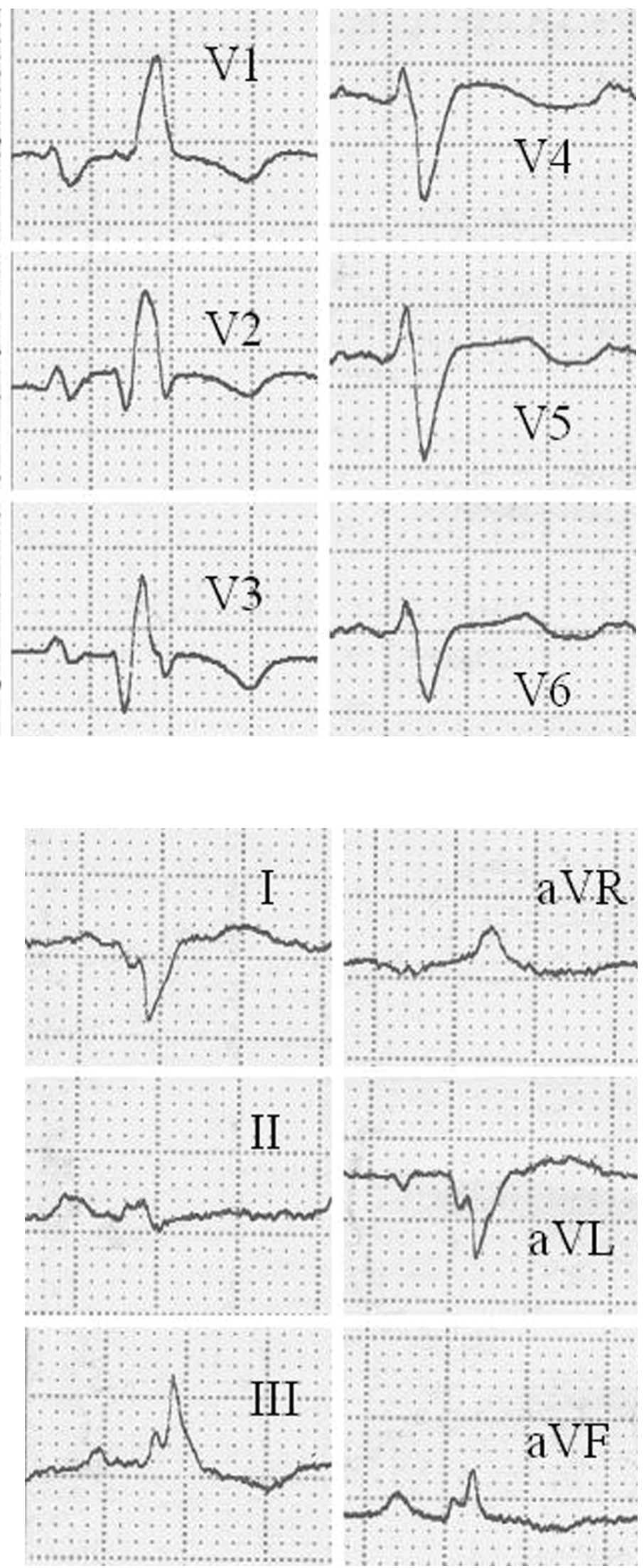

Fig. 2. Ecocardiograma transtorácico. A: Plano paraesternal longitudinal. Importante dilatación de todas las cavidades cardiacas. B: Doppler pulsado del flujo mitral que muestra patrón de llenado de tipo restrictivo. 


\section{TABLA I}

\begin{tabular}{ll} 
Parámetros serológicos & Valores \\
\hline Coxiella burnetti IgG & Negativo \\
Legionella pneumofila & Negativo \\
Anti HBs IgG & Positivo \\
Anti HBc IgM & Negativo \\
Herpes simplex 1 y 2 IgG & Positivo \\
Toxoplasma IgG & Positivo \\
Citomegalovirus IgG & Positivo \\
B. melitensis: Rosa de Bengala e IgG & Negativos \\
VIH 1 y 2 (ELISA) & Negativos \\
Rickettsia conorii y typhi IgG & Negativos \\
\hline
\end{tabular}

$\mathrm{VIH}$ : virus de la inmunodeficiencia humana. HB: virus de la hepatitis $B$. ELISA: análisis de inmunoabsorción ligada a enzimas.

ELISA IgG 2.34) confirmó el diagnóstico de miocardiopatía chagásica crónica. Una endoscopia oral y un enema opaco descartaron patología digestiva chagásica.

La evolución clínica del paciente fue favorable tras tratamiento vasodilatador y deplectivo. Fue dado de alta con tratamiento con furosemida, ramiprilo, carvedilol y acenocumarol. Ha realizado revisiones periódicas en consultas durante el último año, manteniéndose en la actualidad en clase funcional III de la NYHA.

\section{DISCUSIÓN}

En la enfermedad de Chagas, la fase aguda sigue a la primoinfección y es escasamente sintomática. Hasta un tercio de los infectados desarrolla miocardiopatía chagásica crónica, incluso décadas después del contacto inicial. Su patogenia es probablemente multifactorial, incluyendo un efecto directo y continuado del parásito sobre el miocardio, daño del miocito mediado por autoinmunidad y daño del endotelio microvascular por el parásito (4).

Es frecuente encontrar individuos asintomáticos en los que las alteraciones electrocardiográficas (bloqueo de rama derecha con o sin hemibloqueo anterior izquierdo, bradicardia sinusal y distintos grados de bloqueo AV) son su única expresión durante años. Evolutivamente, pueden presentar disfunción autonómica, angor, enfermedad sintomática del sistema de conducción, arritmias ventriculares y muerte súbita. La dilatación biventricular y auricular progresa lentamente hasta la situación de insuficiencia cardiaca congestiva.

En el ecocardiograma, suelen presentar lesiones cicatriciales segmentarias, de modo que más del $50 \%$ de los pacientes sintomáticos presentan aneurismas apicales, que pueden albergar trombos intraventriculares con importante potencial embolígeno. Hasta en un $20 \%$ de los casos, se observan alteraciones segmentarias en la pared postero-inferior del ventrículo izquierdo. Las arterias coronarias son angiográficamente normales, encontrándose incluso ligeramente dilatadas. No obstante, existen importantes alteraciones de la microcirculación (5).

El diagnóstico de la miocardiopatía chagásica crónica se basa en los antecedentes epidemiológicos, los hallazgos clínicos característicos y la positividad de la serología $(6,7)$. El pronóstico es variable, empeorando drásticamente en los pacientes con insuficiencia cardiaca. No se ha demostrado que la eliminación del parásito modifique la evolución de la forma crónica.

La insuficiencia cardiaca se trata con las medidas y fármacos habituales. La amiodarona es ampliamente utilizada en las arritmias ventriculares. El uso de desfibriladores implantables muestra beneficios similares a los obtenidos en enfermos coronarios con muerte súbita, fibrilación ventricular o taquicardia ventricular sostenida. La anticoagulación logra prevenir fenómenos tromboembólicos recurrentes (8). Los receptores de trasplante cardiaco pueden desarrollar infección chagásica aguda en el corazón trasplantado, propiciada por la inmunosupresión. Es necesario el tratamiento pre y postoperatorio con parasiticidas como el benznidazol, mejorando así la supervivencia (9). Es en estos pacientes donde el diagnóstico etiológico de certeza adquiere su mayor importancia.

Se ha revisado la literatura (Medline entre los años 1980 y 2004), encontrándose descrito únicamente un caso de infección chagásica aguda en España en un paciente sometido a trasplante de médula ósea y en el cual no se produjo afectación cardiaca (10). Por lo tanto, el caso presentado constituye el primero en la bibliografía de una miocardiopatía dilatada de etiología chagásica en nuestro país.

Así pues, podemos concluir afirmando que debido a la creciente inmigración de personas procedentes de países donde la enfermedad de Chagas constituye un notable problema sanitario, la miocardiopatía chagásica crónica es una patología que puede ir en aumento en España y que debemos tener presente.

\section{Bibliografía}

1. Manolio TA, Baughman KL, Rodeheffer R, et al. Prevalence and etiology of idiopathic dilated cardiomyopathy (summary of a National Heart, Lung and Blood Institute Workshop). Am J Cardiol 1992; 69: 1054-1061.

2. Moncayo A. Progress towards interruption of transmission of Chagas' disease. Mem Inst Oswaldo Cruz 1999; 94 (Supl. 1): 401-404.

3. Rassi A Jr, Rassi A, Little WC. Chagas' Heart Disease. Clin Cardiol 2000; 23: 883-889.

4. Rossi MA, Bestetti RB. The challenge of chagasic cardiomyopathy. The pathologic roles of autonomic abnormalities, autoimmune mechanisms and microvascular changes, and therapeutic implications. Cardiology 1995; 86: 1-7.

5. Marín-Neto JA, Marzullo P, Marcassa C, Gallo Junior L, Maciel BC, Bellina CR, et al. Myocardial perfusion abnormalities in chronic Chagas' disease as detected by thallium-201 scintigraphy. Am J Cardiol 1992; 69: 780-4.
6. Acquatella H. Avances recientes en miocardiopatía chagásica. Rev Esp Cardiol 1998; 51: 152-157.

7. Girones N, Rodríguez CI, Basso B, Bellón JM, Resino S, Muñoz-Fernández MA, et al. Antibodies to an epitope from the Cha human autoantigen are markers of Chagas' disease. Clin Diang Lab Inmunol 2001; 8: 1039-1043.

8. Herrera RN, Díaz E, Pérez R, Chaín S, Sant-Yacumo R, Rodríguez E, et al. Estado protrombótico en estadios tempranos de la enfermedad de Chagas crónica. Rev Esp Cardiol 2003; 56: 377-382.

9. de Carvalho VB, Sousa EF, Vila JH, da Silva JP, Caiado MR, Araujo SR, et al. Heart transplantation in Chagas' disease. 10 years after the inicial experience. Circulation 1996; 94: 1815-7.

10. Villalba R, Fornés G, Álvarez MA, Román J, Rubio V, Fernández $\mathrm{M}$, et al. Acute Chagas' disease in a recipient of a bone marrow transplant in Spain: case report. Clin Infect Dis 1992; 14: 594595. 